\title{
A Robust Approach for System Identification in the Frequency Domain
}

\author{
Bjørn Gustavsen, Senior Member, IEEE, and Adam Semlyen, Life Fellow, IEEE
}

\begin{abstract}
Accurate modeling of power system components for the purpose of electromagnetic transient calculations requires the frequency dependence of components to be taken into account. In the case of linear components, this can be achieved by identification of a terminal equivalent based on rational functions. This paper addresses the problem of approximating a frequency dependent matrix $H(s)$ with rational functions for the purpose of obtaining a realization in the form of matrices $A, B, C, D$ as used in state equations. It is shown that usage of the Vector Fitting approach leads to a realization in the form of a sum of partial fractions with a residue matrix for each pole. This can be directly converted into a realization in the form $A, B, C, D$ in which $B$ is sparse and each pole is repeated $n$ times with $n$ by $n$ being the size of $H$. The number of repetitions can be strongly reduced and sometimes completely avoided by reducing the rank of the residue matrices, thereby producing a compacted realization which is physically more correct and also permits faster time-domain simulations. The error resulting from the rank-reduction can be reduced by subjecting the realization to a nonlinear least-squares procedure, e.g., Gauss-Newton as was used in this work.
\end{abstract}

Index Terms-Frequency domain fitting, multivariable system, rational approximation, state equation, system identification.

\section{INTRODUCTION}

$\mathbf{O}$ NE difficulty in simulating power system transients is the modeling of components which exhibit frequency dependency in the terminal voltage/current characteristics. Explicit modeling of such components can be very difficult due to the complexity of the underlying phenomena. An alternative approach is black-box modeling, in which a frequency dependent equivalent for the component is identified from measured or calculated frequency responses at its terminals. This technique has been used for the modeling of transmission lines [1], [2], power transformers [3], [4], and for network equivalencing [5]-[7].

The mathematical problem at hand is to identify a terminal model of a linear system based on a given set of frequency domain responses represented by the matrix $H(s)$. This means to identify a model having a frequency response that agrees as closely as possible with $H(s)$, which amounts to finding the matrices $A, B, C, D$ used in the corresponding state equations (state-space model). In addition, the resulting equivalent is required to have only stable poles and to be passive, in order to ensure stable time-domain simulations.

Manuscript received February 8, 2003.

B. Gustavsen is with SINTEF Energy Research, N-7465 Trondheim, Norway (e-mail: bjorn.gustavsen@energy.sintef.no).

A. Semlyen is with the Department of Electrical and Computer Engineering, University of Toronto, Toronto, ON M5S 3G4, Canada (e-mail: adam.semlyen@utoronto.ca).

Digital Object Identifier 10.1109/TPWRD.2003.822530
The authors have developed a powerful method for system identification in the frequency domain by the Vector Fitting approach (VF) [8], [9], which also permits the enforcement of stable poles. Passivity can be enforced by a final correction to the solution [10]. The robustness and public domain availability of VF has lead to its use in diverse applications, including the modeling of power transformers [11], high-speed interconnects [12], and the response of multilayer composite panels to direct lightning strokes [13].

Application of VF to multiterminal systems results in a realization with repeated poles for the columns of $H$ together with a sparse $B$ [7]. This realization is "unusual" because the direct derivation of state equations for physical systems leads to a realization without pole repetitions and with a full $B$. For instance, if the zero-sequence magnetizing dynamics of a transformer is characterized by a single pole, it should appear only once in a partial fraction expansion but with a $3 \times 3$ matrix coefficient (the residue matrix), as shown in Appendix A. This is in contrast to the noncompact realization produced by VF which would get a separate pole for each column of the matrix coefficient. In addition, the compacted form is computationally more efficient in time-domain applications, as shown in Section II-F.

This paper examines the relation between these two alternatives and presents a procedure for converting the realization obtained by VF into compact form. This is achieved by a rank-reduction technique followed by a final optimization by a Newton-type nonlinear least-squares (NLLS) method. Calculated results are shown for two different situations: a case where an exact identification exists, and a case where only an approximate identification can be obtained. The compacting step has been incorporated in an existing public domain computer code for identification of multiterminal systems; see [7].

\section{SYSTEM IDENTIFICATION}

\section{A. State Equations}

The most common realization of linear, time-invariant systems is in the form of matrices $A, B, C, D$ as used in state equations. This realization is obtained by approximating in the least-squares sense the frequency-dependent matrix $H(s)$

$$
H(s)=C(s I-A)^{-1} B+D+s E
$$

where $s=j \omega$. A can practically without loss of generality be assumed to be diagonal (via a similarity transformation). The matrix sizes of an $N$ th order realization of an $n$-terminal system are

$$
\begin{aligned}
& H:(n \times n), C:(n \times N), A:(N \times N) \\
& B:(N \times n), D:(n \times n), E:(n \times n) .
\end{aligned}
$$


It is noted that (1) is nonlinear due the matrix inversion. Therefore, (1) represents an NLLS problem. Its general solution is discussed in the literature, e.g., [14].

\section{B. Residue Matrices}

The identification can also be done using residue matrices $R$, which leads to a least-squares problem of the form

$$
H(s)=\sum_{m=1}^{N}\left(R_{m} \cdot \frac{1}{s-a_{m}}\right)+D+s E .
$$

This problem is also of type NLLS, due to the pole in the denominator. $R_{m}$ is of size $n \times n$.

\section{Conversion to State Equations}

A realization in the form of (2) can be brought to the form (1) by factorizing each term in (2)

$$
R_{m} \cdot \frac{1}{s-a_{m}}=R_{m} \cdot\left(\frac{1}{s-a_{m}} \cdot I_{n}\right) \cdot I_{n}
$$

where $I_{n}$ is an identity matrix of size $n$. The building of $A, B$, $C$ from each term in (2) can then be done as shown in Fig. 1, with $n=3$ and $N=4$.

It is noted that each pole in (2) is repeated $n$ times in $A$. Thus, $A$ is diagonal with $N$ blocks of size $n$. The matrix sizes are

$$
\begin{aligned}
& C:(n N \times N), A:(n N \times n N) \\
& B:(n N \times n), D:(n \times n), E:(n \times n) .
\end{aligned}
$$

Thus, the resulting matrices $A, B, C$ are larger than in (1) due to the repetition of poles in $A$. It is noted that $B$ is sparse.

\section{Compacting}

Unnecessary repetitions of poles can be avoided by considering the relation between (1) and (2). It is noted that each term on the diagonal in (1) multiplies a column in $C$ and a row in $B$. It follows that (1) can be directly converted into (2) where each matrix $R_{m}$ is obtained by multiplying column $m$ of $C$ with row $m$ of $B$. Thus, $R_{m}$ is of rank 1 , implying that it has only one nonzero singular value.

This observation makes it possible to compact the state equation obtained via (2) by expanding each $R_{m}$ into $n$ rank-1 matrices using the singular value decomposition (SVD)

$$
R_{m}=U \Sigma V^{T}
$$

where $\Sigma$ is a diagonal matrix containing the singular values of $R_{m}$ in descending order.

If only the first $r$ leading singular values of a given $R_{m}$ are nonzero, then $R_{m}$ is of rank- $r$ and (4) can be written as a sum of $r$ outer products

$$
R_{m}=\sum_{j=1}^{r} U(:, j) \Sigma(j, j) V^{T}(j,:)=\sum_{j=1}^{r} c_{j} b_{j}^{T} .
$$

It follows that if, for instance, the rank of a given $R_{m}$ equals $r=2$, then two identical poles are produced on the diagonal of $A$ together with two columns in $C$ and two rows in $B$. If $R_{m}$ is rank-1 then only a single pole results on the diagonal, together with a single column in $C$ and a single row in $B$. The latter case is shown in Fig. 2.

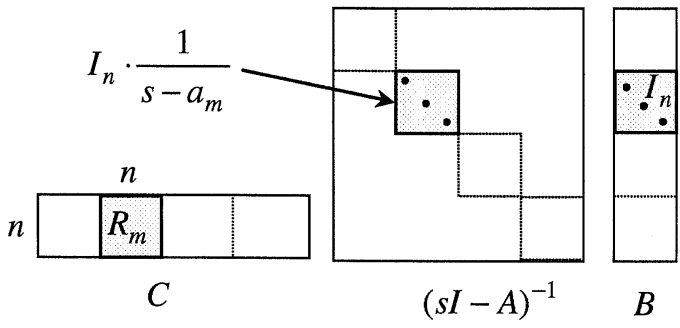

Fig. 1. The contribution from the $m$ th term in (2).

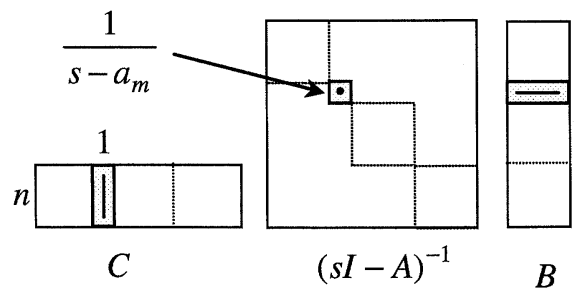

Fig. 2. Contribution from $m$ th term in (2) when $R_{m}$ is rank-1.

Thus, if all $R$-matrices are rank-1, then each $R$-matrix results in a single element on the diagonal in Fig. 2 and the matrix size of $A$ has been reduced from $n N$ to $N$.

\section{E. Optimization}

In many practical situations, the first singular values are much larger than the rest, thus permitting the smallest singular values to be set equal to zero. This implies an approximation of the residue matrices which produces an error in the compacted realization. This error can, however, be reduced by subjecting the realization to an error minimization procedure. Relevant approaches are discussed in [14].

\section{F. Efficiency Considerations}

One justification for the compacting procedure-in addition to leading to the canonical, physically most natural form of state equation realization (see Appendix A for an example)—is that it permits more efficient time-domain simulations. Consider an $n$-terminal system where each column has been fitted with $N$ poles. The computational effort in each time step can be assessed in terms of the number of elementary numerical operations (addition, multiplication). In EMTP-type programs which utilize trapezoidal integration, the convolution with a single pole can be done at the cost of two multiplications and two additions. In addition, the output from the convolution multiplies one column in $C$ which is added to the output vector $y$. For the realization with repeated poles the total computational cost per time step is therefore

$$
C_{1}=n \cdot(4 N+2 n N)=4 n N+2 n^{2} N .
$$

For the compacted realization, the total number of poles is reduced by a factor of $n$. On the other hand, $B$ is now full. This leads to a computational cost

$$
C_{2}=4 N+4 n N \text {. }
$$

When $n \gg 1$, we see that the compacted realization is more efficient by a factor of $n / 2$. Thus, for a six-terminal system, a better than threefold efficiency increase is achieved. 


\section{PRACTICAL IMPLEMENTATION}

\section{A. Realization With Repeated Poles}

VF is a method developed by the authors which solves the least-squares problem stated in (2) by a pole relocation technique. This is achieved by stacking all columns of $H(s)$ into a single column (vector) which is then subjected to approximation with a common set of poles for all matrix elements. The procedure is explained in detail in [8].

VF solves (2) directly without constraints on the rank of the $R$-matrices. This implies that each $R$ is generally of full rank. Therefore, if $N$ starting poles are used (see [8] about starting poles) we get a realization with structure as shown in Fig. 1, where the matrix size of $A$ equals $n N$.

Note that in our previous work, we have interchanged columns in $C$, rows in $B$, and rows and columns in $A$ to produce a realization with a different structure, see $[7$, Sec. IV]. This alternative structure is shown in Fig. 3. $A$ now has a submatrix $A^{\prime}$ of dimension $N$ which is repeated on the diagonal $n$ times, and $B$ consists of $n$ columns of ones. This is what we referred to as "columnwise realization" because each column of $H$ is in effect represented by a separate block in $C$ and in $A$. This realization is, however, equivalent to the one in Fig. 1.

\section{B. Compacting}

After obtaining the realization (2), the $R$-matrices are subjected to rank reduction by inspection of their singular values. Thus, each $R$ is replaced by a rank reduced matrix

$$
R_{m} \approx \sum_{j=1}^{r} U(:, j) \Sigma_{j, j} V^{T}(j,:)
$$

where $r$ denotes the last retained singular value satisfying

$$
\frac{\Sigma_{r, r}}{\Sigma_{1,1}}<\text { tol }
$$

A suitable value for tol can be estimated by considering the required accuracy of the final approximation. The value of tol will need to be adjusted if the desired accuracy is not met.

Finally, a realization in the form of (1) is obtained by forming the outer products (5).

\section{Optimization}

The matrices $A, B, C, D$, and $E$ can be refined by any suitable method for optimization. Appendix B shows details about an implementation based on the Gauss-Newton method.

\section{CAlculated Results}

\section{A. Exact Identification}

In this example we look at a case where the identification can be done without error. The underlying system is rational and of finite order and the frequency domain responses are obtained without measurement noise.

We wish to calculate a compact realization of the type $A$, $B, C, D$ with respect to the sending end of a $10-\mathrm{km}, 24-\mathrm{kV}$ overhead line. The line, which is assumed to be balanced, has a ground fault and is terminated by a resistive load. The line is

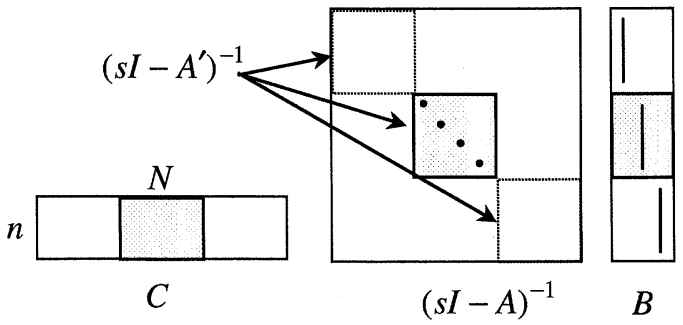

Fig. 3. Columnwise realization of (2).

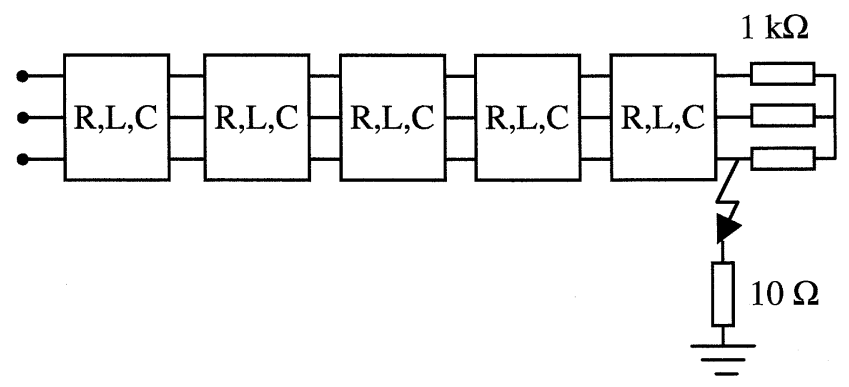

Fig. 4. Distribution overhead line system.

subdivided into five sections where each section is modeled as a coupled PI-circuit with matrices $R, L, C$ calculated at $5 \mathrm{kHz}$ (see Fig. 4). The network is reduced with respect to the terminals at the sending end, giving a 3 by 3 admittance matrix $Y$. Due to the presence of the ground fault at the receiving end, the resulting $Y(s)$ is unbalanced.

Step 1: Realization With Repeated Poles: Fig. 5 shows how the rms-error of the approximation by (2) decreases as the number of poles (terms) in (2) is increased. It is seen that the rms-error decreases abruptly from about $1 \mathrm{e}-5$ to about $1 \mathrm{e}-17$ when increasing the order from 28 poles to 30 poles, which implies that 30 poles is the required order. When converting (2) into a realization of the form (1), the total number of poles increases to 90 because all poles are repeated 3 times $(n=3)$. The elements of $Y$ and their approximation by (1) is shown in Fig. 6 (magnitude functions), as well as the magnitude of the complex deviation.

Step 2: Compacting: In this case (exact identification) all $R$-matrices automatically become of rank-1. By doing the expansion into outer products (5) as described in Section II-D, the total number of poles was reduced from 90 to 30, as the repetition of poles was avoided. The rms-error now became 5.6e-17, which is virtually zero. Thus, the total number of poles has been reduced by a factor of $n=3$ without loss of accuracy!

This is exactly what one could expect by considering that each PI-circuit can be modeled using six state variables (two state variables for each of the three modes). It therefore follows that a state equation should have a total of $5 \times 6=30$ poles.

\section{B. Low-Order Approximation}

In many practical situations, one wishes to obtain a low-order approximation of a high-order system. As an example, we consider the surge admittance matrix $Y_{\mathrm{c}}$ of a three-conductor unbalanced overhead line (phase domain).

Step 1: Realization With Repeated Poles: Fig. 7 shows the nine elements of $Y_{\mathrm{c}}$ as approximated by (2) using eight poles 


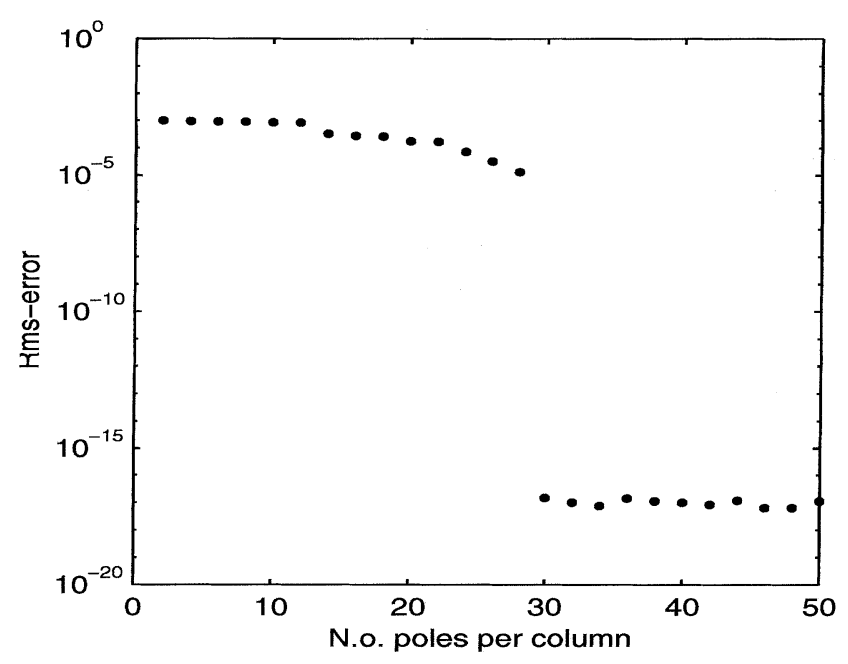

Fig. 5. RMS-error of (2).

(terms). Thus, the direct conversion of (2) into the form (1) leads to a realization with a total of $3 \times 8=24$ poles as the eight poles are repeated three times.

Step 2: Compacting: In order to reduce the order of the realization, we consider removing pole repetitions by compacting. Table I shows the singular values of the eight $R$-matrices in (2). It is seen that $R_{1}, R_{2}$, and $R_{4}$ have a first singular value which is much larger than the subsequent singular values. Thus, one may consider to keep only the first singular value of $R_{1}, R_{2}$, and $R_{4}$ while keeping all singular values of the remaining $R$-matrices. The effect would be a removal of six pole repetitions, giving a realization of $24-6=18$ poles.

Instead, we will investigate the effect of retaining: 1) the first two singular values of all $R$-matrices and 2) only the first singular value of all $R$-matrices. This will result in a realization in the form of (1) with a total of 16 and 8 poles, respectively. The result is shown in Table II. The original full-rank approximation gives an rms-error of 2.11e-6. Approximation with two and one singular values makes the rms-error increase by a factor of 18 and 67 to $3.82 \mathrm{e}-5$ and $1.43 \mathrm{e}-4$, respectively.

Step 3: Newton Improvement: In order to reduce the error, the realization (1) was subjected to a refinement using the Gauss-Newton approach described in Appendix B. Table III shows the resulting error of the approximation after 100 iterations. Comparison with Table II shows that the fitting error is in the case of the rank-2 and rank-1 realizations greatly reduced, although substantially higher than that of the full rank realization. It is also seen that in the case of no compacting (rank $=3$ ), application of Gauss-Newton did not reduce the fitting error.

The importance of a good starting point for the NLLS (GaussNewton) cannot be overemphasized. If one in this example took as initial poles logarithmically distributed real poles and in VF only optimized the residues, then the rms-error became $3.88 \mathrm{e}-5$ (instead of 2.11e-6 in Table II). After reduction to rank-1 and optimization by Gauss-Newton, the error became $6.46 \mathrm{e}-4$ and $6.60 \mathrm{e}-5$, respectively (instead of $1.43 \mathrm{e}-4$ and $2.20 \mathrm{e}-5$ ). Thus, the error of the final approximation has increased by a factor of about $3(6.60 \mathrm{e}-5 / 2.20 \mathrm{e}-5)$.

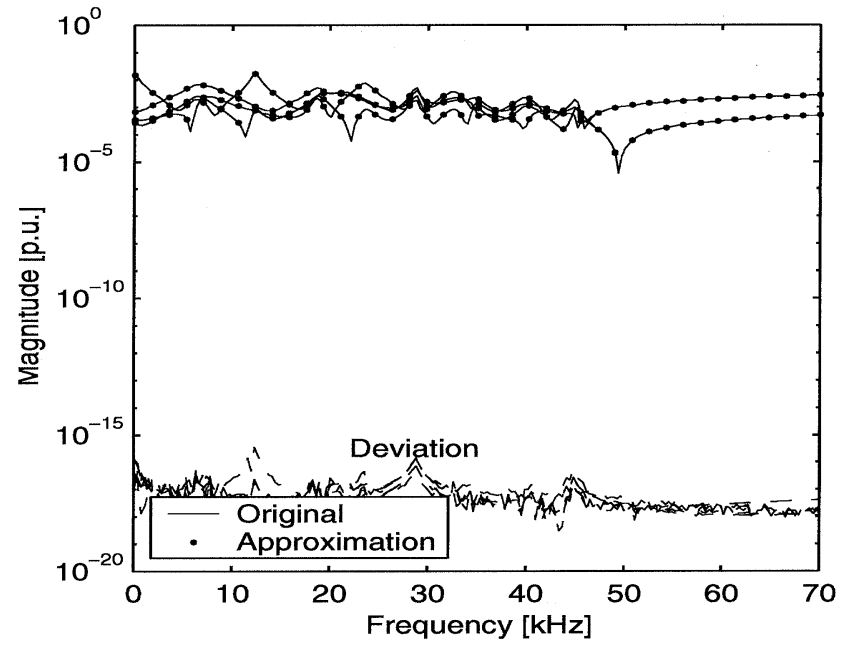

Fig. 6. Approximating $Y(s)$ using 30 poles per column.

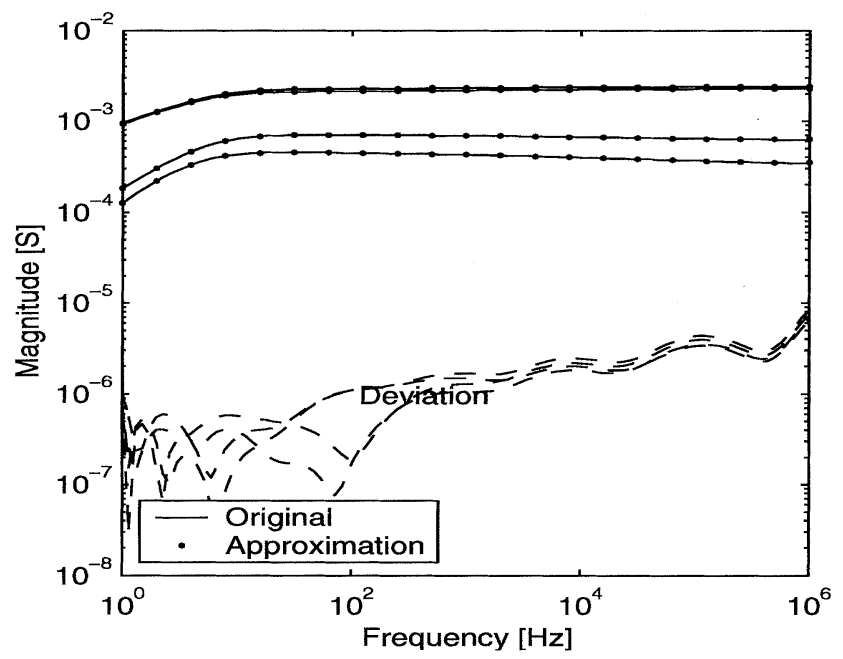

Fig. 7. Approximating $Y_{\mathrm{c}}(s)$ using eight poles per column.

TABLE I

Singular VALUES OF THE $R$-MATRICES IN (2)

\begin{tabular}{l|c|c|c|c|c|c|c|c}
\hline & $R_{1}$ & $R_{2}$ & $R_{3}$ & $R_{4}$ & $R_{5}$ & $R_{6}$ & $R_{7}$ & $R_{8}$ \\
\hline$\sigma_{1}$ & 48.41 & 3.080 & 0.217 & 0.019 & 0.034 & 0.028 & 0.006 & 0.0015 \\
\hline$\sigma_{2}$ & 1.931 & 0.427 & 0.134 & 0.002 & 0.012 & 0.023 & 0.006 & 0.0015 \\
\hline$\sigma_{3}$ & 1.840 & 0.307 & 0.100 & 0.001 & 0.005 & 0.003 & 0.003 & 0.0014 \\
\hline
\end{tabular}

TABLE II

EFFECT OF COMPACTING ON FITTING ERROR

\begin{tabular}{l|c|c|c}
\hline Rank of $R$ & 3 & 2 & 1 \\
\hline rmserr & $2.11 \mathrm{e}-6$ & $3.82 \mathrm{e}-5$ & $1.43 \mathrm{e}-4$ \\
\hline
\end{tabular}

TABLE III

RMSERROR AFTER 100 ITERATIONS

\begin{tabular}{l|c|c|c}
\hline Rank of $R$ & 3 & 2 & 1 \\
\hline rmserr & $2.11 \mathrm{e}-6$ & $1.03 \mathrm{e}-5$ & $2.20 \mathrm{e}-5$ \\
\hline
\end{tabular}




\section{THE NEED FOR REPEATED POLES}

One may ask whether repeated poles in (1) should ever occur for physical systems. While single poles is the normal situation, one can easily find situations where repeated poles will exist.

One important case is when $H(s)$ is a balanced matrix, i.e., all diagonal elements are equal and all off-diagonal elements are also equal. This is the situation that results when one assumes a transmission line to be continuously transposed. It can then be shown that all $R$-matrices of a finite order system are of rank-2, which means that all poles in the realization (2) are repeated two times.

One can also find situations where all poles are repeated $n$ times so that compacting is not possible and all $R$-matrices are of full rank. Consider, for example, an electrical network with given nodes and branches between all nodes and between all nodes and ground. If all branches have the same pole (but possibly different residues) then the $R$-matrix of the admittance matrix will have full rank. By connecting such branches in parallel, one can obtain a network where all $R$-matrices of $Y$ are of full rank. This is the type of electrical network that resulted when creating an equivalent electrical network as described in [7].

\section{DISCUSSION}

The paper has shown that rational approximation of a matrix $H(s)$ of dimension $n$ using $\mathrm{VF}$ is equivalent to approximating $H(s)$ by a sum of $R$-matrices, each multiplied by a single-pole fraction [see (2)]. The solution can be converted into a "standard" realization $A, B, C, D$, as in (1), in which the poles are repeated $n$ times. By considering the rank of the $R$-matrices, some or all of the repetitions can be avoided, thus leading to a more compact realization which is physically more correct. It was demonstrated that this compacting of the realization does not involve any errors provided that the fitting is done with zero error. However, it was shown that when a fitting error exists (which is the normal situation), the $R$-matrices are in general of full rank, so that compacting will increase the fitting error. The error can, however, be reduced by subjecting the obtained realization $(A, B, C, D)$ to optimization by any effective NLLS method. Application of Gauss-Newton was found to strongly reduce the fitting error. However, we believe that a more carefully implemented NLLS might be devised so as to reach the true minimum. Thus, for instance, it was noted in the example with $Y_{\mathrm{c}}$ that the Jacobian (B.1) was ill-conditioned, which implies an inaccurate search direction. Also, the search may have become stuck in a local minimum. The final optimization step could therefore be a worthwhile topic for future research.

It was shown in Section III-C that compacting can be expected to increase the efficiency by a factor $n / 2$. For the example with exact identification (Section IV-A), we had $n=3$, which means a $50 \%$ increase in the efficiency. The potential speed increase can be of importance in some applications, e.g., Monte Carlo simulations. For situations with a low-order approximation (e.g., Section IV-B) one has to take into account that the compacting increases the fitting error significantly, which means that one has to increase the order of approximation to maintain the same error. Again, a more sophisticated optimization procedure may reduce the error.

\section{CONCLUSIONS}

The problem considered is the approximation of a frequencydependent matrix $H(s)$ of dimension $n$ by $n$ by a rational function. This paper has investigated the relation between a traditional realization of the form $A, B, C, D$ with compact matrices and the realization that is produced by VF. The main conclusions are as follows.

- VF leads to a realization in the form of a sum of partial fractions with a residue matrix $R$ for each pole. This realization can be directly converted into a realization of the form $A, B, C, D$ by expanding each $R$-matrix into a sum of rank-1 matrices. The resulting realization has a sparse $B$ and each pole is repeated $n$ times where $n$ is the size of $H(s)$.

- The number of repetitions can be reduced or avoided by considering the rank of the $R$-matrices. In the case that the approximation is exact (finite-order system and no noise in $H$ ), all $R$-matrices become rank- 1 and can thus be expressed by outer products which define the columns and rows in $C$ and $B$ of a compact realization without pole repetitions. Some repetitions may still be needed in special cases, e.g., in the case of a balanced $H$.

- Compacting causes the computational efficiency in the time domain to be increased by a factor of about $n / 2$, where $n$ is the number of terminals. This assumes trapezoidal integration.

- When the approximation is not exact, the $R$-matrices are in general of full rank. The number of pole repetitions can be reduced by rank-reduction of each $R$ by discarding the contribution from the smallest singular values. This increases the error of the approximation. The error can, however, be reduced by subjecting the resulting realization $(A, B, C, D)$ to optimization by a general nonlinear least-squares procedure, e.g., Gauss-Newton. More sophisticated optimization is a subject for further research.

- In general, VF can be viewed as an approach for obtaining a very good initial solution for iterative nonlinear leastsquares methods which aim at finding an optimum compact realization of the form $A, B, C, D$ of a given order.

- The compacting step has been incorporated in a public domain software [7] for identification of multiterminal systems.

\section{APPENDIX A \\ EXAMPLE OF COMPACT REALIZATION}

Consider a simple low frequency model of a three-phase transformer represented by a number of discrete windings. Then the variables are: flux linkages $\psi$, voltages $v$, and currents $i$, related via

$$
\begin{aligned}
\dot{\psi} & =-R i+v \\
i & =M \psi
\end{aligned}
$$

where $M=L^{-1}$. These give

$$
\dot{\psi}=-R M \psi+v
$$

which, together with (A.2), are exactly of the form of compact linear state equation realization with coefficients $A, B$, and $C$. 
The essential feature of the obtained compact form is that it arose naturally from physical reasoning and it is formally not different from the single input and output case. If $A$ is a scalar $a$ (and correspondingly $B=b^{T}, C=c$ ), then we get the transfer function

$$
H(s)=\frac{c b^{T}}{s-a}=\frac{R}{s-a}
$$

and we see that the residue matrix $R$ is of rank one. More generally, a rank-one residue matrix will result for each eigenvalue of $A$, repeated ones included. In this latter case, the rank of the resultant $R$ will equal the multiplicity of the eigenvalue.

\section{APPENDIX B \\ GAUSS-NEWTON IMPROVEMENT}

\section{A. Gauss-Newton}

The following outlines an implementation of the Gauss-Newton method [14] for finding an optimized approximation of a realization in the form $A, B, C, D$. To reduce the least-squares error, a correction to $A, B, C$ is calculated via the Jacobian matrix $J$. The columns of $J$ correspond to the elements of $A, B, C$ as follows:

$$
\left[\begin{array}{lll}
J_{A} & J_{B} & J_{C}
\end{array}\right]\left[\begin{array}{l}
\Delta x_{A} \\
\Delta x_{B} \\
\Delta x_{C}
\end{array}\right]=b
$$

where $b$ is the error.

The step calculated by Gauss-Newton is

$$
\Delta x=\left(J^{T} J\right)^{-1} J^{T} b .
$$

In situations where the Jacobian is ill-conditioned, we use instead the "perturbed Newton" [14, p. 151]

$$
\Delta x=H^{-1} J^{T} b
$$

where

$$
H=J^{T} J+\sqrt{n \cdot \text { macheps }} \cdot\left\|J^{T} J\right\|_{1} \cdot I .
$$

$n$ is the number of elements of $\Delta x, I$ is the identity matrix, and "macheps" is the machine precision.

If the step $\Delta x$ causes the error to increase, $\Delta x$ is replaced with $0.5 \Delta x$ repeatedly until the error is reduced. This step reduction is necessary in order to ensure convergence of the minimization process.

\section{B. Normalization}

The Jacobian matrix $J$ will in general be singular due to an arbitrariness in the solution, as the scaling of the columns of $C$ and rows of $B$ is not uniquely defined. This problem is overcome by removing from the Jacobian columns which correspond to the elements of the first column of $B$.

\section{Symmetry}

When the transfer matrix $H(s)$ is symmetric, the columns of $C$ (in the compact form) become related to the rows of $B$ by a constant

$$
C_{j}=k B_{j}^{T} .
$$

Thus, by rescaling the columns of $C$ and the rows of $B$, we achieve

$$
C=B^{T} .
$$

It now follows that $\Delta x_{B}=\Delta x_{C}$ in (B.1), which can be rewritten into

$$
\left[\begin{array}{ll}
J_{A} & J_{B}+J_{C}
\end{array}\right]\left[\begin{array}{l}
\Delta x_{A} \\
\Delta x_{B}
\end{array}\right]=b .
$$

Normalization is not needed in the symmetrical case.

\section{Complex Conjugacy}

Complex conjugacy of the poles is ensured by including negative frequencies in the Newton process

$$
H(s)=H^{*}\left(s^{*}\right) .
$$

\section{REFERENCES}

[1] J. R. Marti, "Accurate modeling of frequency-dependent transmission lines in electromagnetic transient simulations," IEEE Trans. Power App. Syst., vol. PAS-101, pp. 147-156, Jan. 1982.

[2] A. Morched, B. Gustavsen, and M. Tartibi, "A universal line model for accurate calculation of electromagnetic transients on overhead lines and underground cables," IEEE Trans. Power Delivery, vol. 14, pp. 1032-1038, July 1999.

[3] A. Morched, L. Marti, and J. Ottevangers, "A high frequency transformer model for the EMTP," IEEE Trans. Power Delivery, vol. 8, pp. 1615-1626, July 1993.

[4] B. Gustavsen, "Wide band modeling of power transformers," IEEE Trans. Power Delivery, to be published.

[5] A. S. Morched, J. H. Ottevangers, and L. Marti, "Multi-port frequencydependent network equivalents for the EMTP," IEEE Trans. Power Delivery, vol. 8, pp. 1402-1412, July 1993.

[6] M. Kizilcay, "Low-order network equivalents for electromagnetic transients studies," Eur. Trans. Elect. Power (ETEP), vol. 3, no. 2, pp. 123-129, Mar./Apr. 1993.

[7] B. Gustavsen, "Computer code for rational approximation of admittance matrices," IEEE Trans. Power Delivery, vol. 17, pp. 1093-1098, Oct. 2002.

[8] B. Gustavsen and A. Semlyen, "Rational approximation of frequency domain responses by vector fitting," IEEE Trans. Power Delivery, vol. 14, pp. 1052-1061, July 1999.

[9] A. Semlyen and B. Gustavsen, "Vector fitting by pole relocation for the state equation approximation of nonrational transfer matrices," Circuits Syst. Signal Process., vol. 19, no. 6, pp. 549-566, 2000.

[10] B. Gustavsen and A. Semlyen, "Enforcing passivity for admittance matrices approximated by rational functions," IEEE Trans. Power Syst., vol. 16, pp. 97-104, Feb. 2001.

[11] M. J. Manyahi and R. Thottappillil, "Transfer of lightning transients through distribution transformer circuits," in Proc. 26th Int. Conf. Lightning Protection (ICLP), Cracow, Poland, Sept. 2002, pp. 435-440.

[12] K. M. C. Branch, J. Morsey, A. C. Cangellaris, and A. E. Ruehli, "Physically consistent transmission line models for high-speed interconnects in lossy dielectrics," IEEE Trans. Adv. Packag., vol. 25, pp. 129-135, May 2002.

[13] M. S. Sarto, "Sub-cell model of multilayer composite materials for full FDTD and hybrid MFIE/FDTD analyses," in IEEE Symp. Electromagnetic Compatibility, 2002, pp. 737-742.

[14] J. E. Dennis Jr. and R. B. Schnabel, "Numerical methods for unconstrained optimization and nonlinear equations," in Proceedings of SIAM. Philadelphia, PA: SIAM, 1996. 
Bjørn Gustavsen (M'94-SM'03) was born in Harstad, Norway, in 1965. He received the M.Sc. and Dr.-Ing. degrees in 1989 and 1993, respectively, from the Norwegian Institute of Technology, Trondheim.

Currently, he is with SINTEF Energy Research, Trondheim. He spent 1996 as a Visiting Researcher at the University of Toronto, ON, Canada, and the summer of 1998 at the Manitoba HVDC Research Centre, Winnipeg, MB, Canada. He was a Marie Curie Fellow at the University of Stuttgart, Germany, in August 2001-August 2002. His interests include simulation of electromagnetic transients and modeling of frequency-dependent effects.
Adam Semlyen (SM'70-F'88-LF'97) was born in Rumania in 1923, where he received the Dipl.Ing. and $\mathrm{Ph} . \mathrm{D}$. degrees.

He was previously affiliated with an electric power utility and held academic positions at the Polytechnic Institute of Timisoara. In 1969, he joined the University of Toronto, Toronto, ON, Canada, where he is a Professor in the Department of Electrical and Computer Engineering (Emeritus since 1988). His research interests include steady-state and dynamic analysis, as well as computation of electromagnetic transients in power systems. 